\title{
Relaciones mutuas entre el embarazo y la enfermedad de Hodking ${ }^{(*)}$
}

\author{
Doctor Mario Gaitán Yanguas \\ Director del Instituto Nacional de Cancerología. Bogotá.
}

Es de concenso unánime en medicina que la evolución del cán. cer se agrava cuando se presenta un embarazo en el curso de su evolución. Esta influencia es especialmente notoria en algunas formas de cáncer, como el del pecho y el del útero, menos acentua. da en otros, como el del estómago, y poco o nada notoria en otros, como el de piel, laringe, etc. Sin embargo, aunque esta opinión se encuentra expresada en forma más o menos dogmática en los li_ bros de texto y en algunas publicaciones, no se encuentran en la literatura médica estudios analíticos comparativos que permitan basar estas afirmaciones en algo sólido y comprobado o diferente cie la simple "impresión", sino únicamente quizá en lo relativo al cáncer del seno.

En cuanto al grupo de los linfomas malignos (linfosarcoma, Hodgkin, leucemias, etc.), se ha dicho también desde hace mucho tiempo que el embarazo es un latigazo que se dá a la enfermedad y que por lo tanto debe evitarse o, si ya se ha presentado, que de. be impedirse su evolución y evitar que llegue a término provocan_ clo el aborto o el parto prematuros, para salvar así la vida de la madre.

En forma análoga se dice también que cuando se presenta el embarazo en una paciente que sufre de un linfoma, el feto está en mucho mayor peligro, pues por una parte la frecuencia de abortos y partos prematuros espontáneos es muy grande, y por otra parte también es muy grande la posibilidad de que se herede la "predisposición al cáncer" y en especial a los linfomas.

(*) Trabajo presentado por el autor al III Congreso de Obstetricia y Ginecología reunido en Ibagué. 
Fácilmente se comprende la extraordinaria importancia de es. tas consideraciones, especialmente en nuestro medio, donde las creencias religiosas de médicos y pacientes, en particular en lo relativo al aborto terapéutico, hacen que la elección de una con_ ducta de tratamiento adquiera matices verdaderamente escabro_ sos y peligrosos para la tranquilidad moral y para la situación jurídica del médico.

Fue por este motivo que resolvimos consultar la literatura médica a nuestro alcance sobre este tema, a la vez que revisar los casos del Instituto Nacional de Cancerología, para ver sí nos era posible llegar a un conocimiento más o menos completo y exacto de la materia, que nos permita formar una opinión justa. En esta forma creemos que podremos prestar alguna ayuda al obstetra que, aunque no muy frecuentemente por fortuna, se encuentre ante esta situación.

El número de casos de concomitancia de enfermedad de Hodgkin y embarazo es bastante bajo en todas partes, debido a cuatro factores principales: 1) Los linfomas en general y el Hodgkin en particular, son formas de cáncer poco frecuentes en los medios hospitalarios extranjeros, al menos más raros que los cánceres de vías digestivas, útero, seno, piel: pulmón, etc. En nuestro medio es un poco más común, pues, como lo hemos mostrado en nuestra trabajo sobre el tema (13), los linfomas ocupan el cuarto puesto de frecuencia entre todos los cánceres y a su vez el Hodgkin re. presenta el $34 \%$ de los linfoblastomas.

2) El Hodgkin es menos frecuente en la mujer que en el hom. bre, en la proporción de una por 2,5 hombres.

3) El Hodgkin se presenta con menos frecuencia durante el período de fertilidad femenina que otros cánceres. Por ejemplo en nuestro medio solo el $64 \%$ de las mujeres estaban en este período.

4) Por lo general la evolución del Hodgkin es grave, en mu. chos casos muy rápida, y altera en alto grado el estado general, lo que hace menos factible un embarazo que en otro tipo de cán. cer como lengua, piel, etc.

Así, Fabian (4) en su tesis hecha en 1943 apenas logra reu. nir 40 casos relatados por 20 autores diferentes. Bichel (1) en 1950 llega a reunir 57 casos, Riva y Col. (9) en 1953 suben a 65 encon. trados en la literatura de los Estados Unidos. Hultberg (7) en 1954 revisa 1.13 casos de la literatura mundial.

Entre nosotros, entre un total de 253 casos de Hodgkin que hemos revisado había 71: mujeres; de ellas 46 estaban en adad de concebir, es decir entre dieciseis y quince años. $Y$ encontramos 7 
casos de embarazo, aunque sospechamos que debió haber varios más pero que no aparecen registrados en las historias clínicas a causa de la deficiencia de' algunas de ellas y de los controles. Cada uno de estos 7 casos se presentó en una mujer distinta, o sea que no encontramos que alguna de ellas hubiera hecho dos o más em. barazos, como sí lo anotan autores como Gilbert (2), quien entre 8 madres encontro 3 que tuvieron 2 embarazos y una que tuvo 3 , o sea un total de 13 embarazos en las 8 madres.

Tres son los puntos importantes por considerar: 1) ¿Agrava el embarazo la evolución de la enfermedad de Hodgkin 2) ¿La en. fermedad de Hodgkin altera el curso del embarazo? 3) ¿Los hi_ jos de mujeres con Hodgkin tienen algunas condiciones de infe_ rioridad en relación con los de mujeres sanas? Consideremos los dos primeros interrogantes en conjunto, dejando para el final el tercero.

La referencia más antigua que pudimos encontrar es el comentario que hace Gilbert (4) a la tesis de Fabian publicada en 1943. Este autor analiza los datos de 20 publicaciones anteriores con 40 embarazos relatados; todas ellas traen casos aislados o en pequeño número, lo que no permite un análisis estadístico ade. cuado; sin embargo Fabian llega a la conclusión de que el emba_ razo sí agrava la evolución del Hodgkin y aconseja su interrup. ción, tal como se hizo en 2 de 3 de sus enfermas que se agravaron.

La misma conclusión sacaría el lector de los casos descritos por Fortmann (8'): una mujer que llevaba nueve años en buenas condiciones después del tratamiento para su Hodgkin quedó em. barazada, tuvo recaída de la enfermedad y murió inmediatamen. te después del parto; otra de sus pacientes se agravó durante el embarazo y murió antes del término; en otra caso el Hodgkin co_ menzó a evolucionar al tiempo con el embarazo, fue tratada, tu. yo su parto normal, y quedó en buenas condiciones hasta 3 años después cuando durante' un segundo embarazo tuvo recaída del Hodgkin, pero alcanzó a completar su embarazo normalmente. En cambio en algunos otros die sus casos no se presentó complicación. de modo que el autor no osa emitir conclusión definitiva.

En 1950 Bichel publica un trabajo muy interesante en que re. visa 65 embarazos que se presentaron en 57 mujeres con Hodgkin: 46 recogidas de la literatura médica y 11 de su casuística perso_ nal. Y llega a la conclusión de que el embarazo no obra desfavora. blemente sobre la evolución de la enfermedad de Hodgkin, a pe. sar die que algunos de estos casos tuvieron agravación simultá_ nea con el embarazo, pero que posiblemente se hubiera presenta_ 
do lo mismo sin él. En cambio parece que el Hodgkin sí compli_ ca la evolución del embarazo, como lo demuestra el hecho de que sólo 42 embarazos llegaron a término con parto normal, o sea $64 \%$. como se ve a continuación:

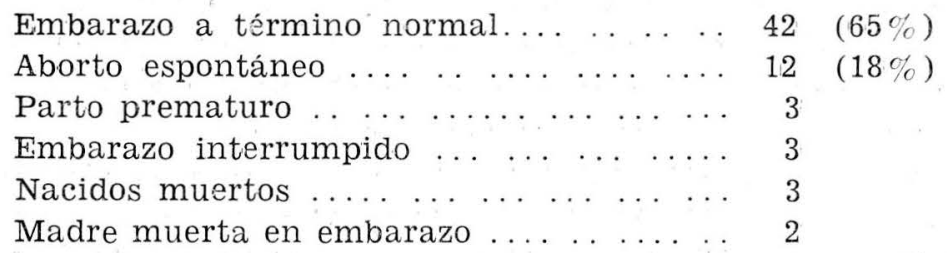

En cambio Riva y sus colaboradores, que analizan 65 casos de esta asociación encontrados en la literatura norteamericana más 8 casos suyos del Hospital Walter Roed, concluyen su estudio diciendo que el Hodgkin no altera la placenta, ni produce hemo. rragias, ni aumenta la frecuencia del aborto espontáneo, y en ge. neral no complica en nada el embarazo, como tampoco éste agra. va la evolución de la enfermedad de Hodgkin.

La misma opinión emiten Hultberg; basándose en 11.3 casos (100 de la literatura y 13 del Radiumhemet), y Teneblat y col. (11) con base en 33 casos ( 32 de la literatura y 1 propio).

En cambio Hertz anota que de 10 casos suyos hubo notoria agravación en 5, o sea la mitad, y es por lo tanto partidario de la interrupción del embarazo.

Como lo dijimos antes, nosotros encontramos 7 casos de em. barazo entre 46 pacientes con diagnóstico de enfermedades de Hodgkin en periodo de fertilidad; en 2 de ellas el embarazo se pre_ sentó durante el primer ataque del Hodgkin y en las otras 5 des. pués de haber sido tratadas la primera vez y haber obtenido su mejoría.

Los 2 casos en que el embarazo apareció durante el período activo del Hodgkin fueron el uno en una mujer de veintitrés años en quien el embarazo comenzó cuando la enfermedad llevaba un mes de evolución aparente; la paciente llegó al Instituto en el sépti_ mo mes de embarazo y no alcanzó a ser tratada porque mientras se le hacían los exámenes clínicos y paraclínicos de rigor presen. tó un parto prematuro con feto muerto y murió a los 12 días de la primera consulta; desafortunadamente no pudo hacerse la aú. topsia de la madre ni del feto. El otro caso fue en una mujer de veinte años que llegó con enfermedad de Hodgkin en sstado III: ganglios múltiples en ambas ingles y en a mbas axilas, se le comen. 
zó el tratamiento con rayos $\mathrm{X}$ en forma ambulatoria y quedó emba. razada en el curso de él; el tratamiento se completó satisfactoria_ mente con desaparición de los ganglios, pero 2 meses después re. gresó la paciente con ganglios mediastinales (que no tenía an_ tes); se le hizo nueva roentgenterapia, sin complicaciones, y el ambarazo siguió su curso normal. Se recuperó por completo de la enfermedad, pero a los 8.5 meses de embarazo presentó sin causa aparente parto prematuro, con hijo vivo, y desde entonces se agra. vó de nuevo para morir un mes más tarde por generalización de la enfermedad; el niño seguía bien hasta los dos años de edad.

En los otros 5 casos el embarazo se presentó durante períocos de inactividad del Hodgkin, es decir, en paciente que, habien. do sido tratadas antes, estaban en ese momento sin manifesta_ ciones aparentes de la enfermedad. En 2 casos el embarazo se presentó a los 3 y 8 meses del tratamiento, en otros 2 entre 1 y 2 años y en el otro a los 4 años.

En el caso número 3 el embarazo comenzó a los 3 meses de haber terminado el tratamiento; era una mujer de treinta y seis años, con 4 hijos, que había llegado con enfermedad en estado II (ganglios cervicales bilaterales); la misma paciente provocó su aborto a los 3.5 meses del embarazo, no presentó signos de recaída de la enfermedad y fue perdida de vista.

El caso número 4 era una mujer de treinta años, con 2 emba. razos antes de que se presentará la enfermedad; llegó en estado III, con ganglios en ambos lados del cuello y en región axilar iz. guierda. Fue sometida a tratamiento con recuperación completa. A los \& meses quedó embarazada y más o menos a partir del sexto mes de embarazo comenzó a presentar ganglios en axila derecha y ambas ingles, pero la paciente no vino a tratamiento sino des. pués de haber tenido su parto normal con hijo vivo. Después del segundo tratamiente sobrevivió 4 meses, habiendo muerto por generalización del mal. Se ignora el estado actual del hijo.

El caso número 5 , en mujer también de treinta años, con 4 embarazos anteriores normales, llegó en estado II: ganglios en ambos lados del cuello. A los 19 meses de su tratamiento exitoso quedó embarazada y tuvo curso normal hasta el quinto mes en que aparecieron ganglios mediastinales, esplenomegalia y dudosos ganglios axilares. Solo volvió a tratamiento después de su parto normal con hijo vivo; se la sometió a roentgenterapia y tuvo remisión de 11 meses, al cabo de los cuales hizo generalización más o menos súbita y murió a los 13 meses de este segundo tratamien. to. Tampoco se conoce la situación actual del hijo. 
El caso número 6 era de una joven de diecinueve años que llegó con la enfermedad en estado III: ganglios cervicales y axi. lares bilaterales y dudosos en mediastino. Se le hizo roentgentera. pia con recuperación, y a los 11 meses quedó embarazada. El em. barazo pasó normalmente, el parto fue normal así como el hijo y no presentó recaída del Hodgkin, pero desafortunadamente se perdió de vista.

El caso número 7 fue en una mujer, de veintiocho años, con 2 embarazos antes de su enfermedad; llegó en estado I, con ganglios en el lado derecho del cuello. Se le hizo la extirpación de ellos, en seguida se le dio tratamiento con rayos $\mathrm{X}$ y quedó en buenas condiciones. Cuatro años más tarde tuvo un embarazo normal con parto normal y con hijo vivo, del cual se sabe que estaba bien hasta 2.5 años después

$\mathrm{O}$ sea que, resumiendo, tenemos 2 casos en que coexistieron el embarazo y el Hodgkin en período de evolución y en ambos la en. termedad evolucionó en forma muy desfavorable y grave y el em. barazo también fue alterado en su curso. Y tenemos 5 casos en que el embarazo se presentó durante períodos inactivos del Hodgkin. Uno de ellos en estada I, con evolución muy satisfactoria pa. ra ambas entidades. Dos casos de estado II de los cuales uno mu. rió a los 13 meses del parto y la otra, que provocó su aborto, que se perdió de vista. Y 2 en estado III, de las cuales una sobrevivió 4 meses al parto y la otra que se perdió de vista.

Con base en estos siete casos solo podríamos decir, sin que sea una opinión definitiva, pues el número de enfermas es muy limitado, que la asociación simultánea de Hodgkin evolutivo y embarazo es desfavorable para ambas entidades. En cuanto a los otros cinco casos, no se puede afirmar nada, pues el número de pacientes con evolución desfavorable del Hodgkin bien puede co_ rresponder a lo que sucede con cualquier grupo de pacientes aún no embarazadas y aún con los hombres.

Pero hay en la literatura un trabajo muy bien realizado, por Southam y col. (10), a fines de 1956, que presenta conclusiones estadísticas muy valederas al respecto. Estos autores revisaron to. dos los casos del Memorial Hospital y del Sloane Hospital for Wo. men y reunieron 59 pacientes con 83 embarazos durante la evolu. ción del Hodgkin. En 26 de ellas el Hodgkin principió su desarro. 110 durante algún embarazo y en 33 el embarazo se presentó des_ pués de haber sido tratadas para el Hodgkin, estando 23 de éstas con signos de que la enfermedad no estaba definitivamente con. trolada y 10 sin ninguna evidencia de enfermedad activa. Los 
promedios de supervivencia encontrados en estos tres grupos fue_ ron los siguientes:

$\begin{array}{cr}\begin{array}{c}N^{\circ} \text { de } \\ \text { casos }\end{array} & \begin{array}{r}\text { Promedio de } \\ \text { supervivencia } \\ \text { Meses }\end{array} \\ 26 & 46.0 \\ 23 & 75.0 \\ 10 & 128.3\end{array}$

Los cálculos estadísticos del "promedio de error standard" (7.6, 14.5 y 21.9 respectivamente para cada grupo), de la desvia_ ción standard $(38,68$ y 70 respectivamente para cada grupo), de la "relación crítica", etc., indican que estos datos son valederos y significativos.

Hay además otro dato muy importante en este trabajo: es el relato de dos enfermas en quienes se observó agravación muy rápida del Hodgkin durante el embarazo y que fueron sometidas al aborto terapéutico, mostrando "regresión parcial espontánea" de los tumores después del abortó.

En cuanto al efecto del Hodgkin sobre el embarazo, Southam y sus colaboradores, después de analizar los 83 embarazos descritos y de compararlos con otros 50 que habían tenido antes cuan. do sanas, 21 de estas mismas mujeres concluyen diciendo, que "la presencia de Hodgkin diseminado, o de localización abdominal, o la evidencia de Hodgkin activo en cualquier sitio, aumenta las po_ sibilidades de perjuicio para el embarazo".

Las conclusiones derivadas de este magnífico trabajo son, ca_ si pudiera decirse, iguales a las ya enunciadas por Gilbert en 1951 (3) en la siguiente forma: 1) No debe interrumpirse el embarazo en las mujeres que hayan sido tratadas con éxito para formas de evolución lenta del Hodgkin. 2) Si el embarazo se presenta duran. te períodos de actividad del Hodgkin, o si éste aparece durante el embarazo, hay perjuicio para las dos entidades y debe por lo tan. to hacerse un tratamiento muy cuidadoso.

Influencia sobre el hijo.-Entre las 7 enfermas que estudia. mos hubo un aborto provocado y dos partos prematuros sin que hubiera podido hacerse el estudio anatomo_patológico del feto. En las otras 4 enfermas con parto normal, los hijos no mostraron evidencia clínica de la enfermedad, pero desafortunadamente 3 se 
perdieron de vista. Sólo quedan así dos hijos con evolución cono_ cida: uno de parto prematuro que se sabe estaba bien 2 años des. pués y otro de parto normal que estaba bien a los 2.5 años.

En la literatura médica colombiana solo conocemos la publi_ cación de Otero (12) sobre ocurrencia familiar de linfoblastomas; este autor informa sobre dos familias: en la primera, dos niños hermanos tuvieron simultáneamente enfermedad de Hodgkin y poco después su padre presentó un linfosarcoma linfoblástico, y en la otra, una mujer de veinticinco años, su tío paterno y un pri_ mo paterno presentaron respectivamente linfosarcoma giganto_fo. licular, Hodgkin y linfosarcoma reticular no simultáneamente. Pe. ro no hemos encontrado ningún informe que relacione la enfer. medad de Hodgkin de un paciente con la misma enfermedad en su madre.

Entre los casos de mujeres hodgkinianas informados por auto_ res extranjeros tampoco se relata que sus hijos hayan tenido la enfermedad, ni en los 73 partos analizados por Riva y col, ni en. entre los 13 casos de Hultberg (el mayor de los cuales tenía ya quince años), ni entre los 32 de Teneblat y col., ni entre los 65 partos estudiados por Bichel, ni entre los 13 casos de Gilbert, el mayor de los cuales llegaba ya a los veinticuatro años; inclusive Bichel hizo cortes seriados de la placenta y de los fetos de abor. tos sin encontrar muestra alguna de enfermedad de Hodgkin.

En cambio en la serie estudiada por Southam y col. de 83 embarazos, se relatan algunas anomalías; de estos 83 embarazos se sabe que 9 hijos estaban bien entre los seis y doce meses después del nacimiento; otros 20 , entre uno y cuatro años; 6 , entre cinco y diez años, y 2 , de once y catorce años, respectivamente. Pero anotan un caso que nació con anomalías congénitas y otro que al año del nacimiento presentó un adenocarcinoma anaplás_ tico. Aunque estos autores afirman que no es posible atribuir es. tas anormalidades a la enfermedad de Hodgkin, es lástima que no aclaren si ellas correspondían a enfermas que tenían localización abdominal y que fueron irradiadas, o si eran enfermas de otras cocalizaciones, o si recibieron tratamientos con alguna de las dro. gas citotóxicas, pues cualquiera de estas circunstancias podría es_ tar relacionada con la anormalidad.

Comentario-Presentamos 7 casos de embarazo en mujeres con enfermedad de Hodgkin, 2 de los cuales ocurrieron durante períodos de actividad del mal y los otros 5 en períodos de inactivi. dad después del tratamiento. En el primer grupo la evolución fue muy desfavorable para el Hodgkin y para el embarazo y por lo 
tanto para el feto. En el grupo restante no hubo una relación de. finida que permita llegar a conclusiones positivas.

Nos parece de extraordinaria trascendencia definir las rela_ ciones mutuas entre el Hodgkin y el embarazo, pues de ello depen. de la conducta que deban tomar el médico internista por una parte y el obstetra por la otra, ante una enferma en estas condicio. nes. Si debe desaconsejarse o prohibirse el embarazo en las pa. cientes hodgkinianas; si una vez presente el embarazo debe acon. sejarse interrupción o no; si hay peligros de que el hijo concebido en estas circunstancias quede en condiciones de inferioridad más expuesto a anomalías o a la aparición de entidades cancerosas durante su vida.

Como con base en nuestros 7 casos no podemos hacer afirmaciones al respecto, estudiamos alrededor de unos doscientos o más casos que encontramos en la literatura médica mundial, lo que nos permite definir las siguientes conclusiones:

1) Debe desaconsejarse el embarazo en mujeres que estén en periodo de evolución activa del Hodgkin.

2) Cuando el Hodgkin comienza a evolucionar durante el em. barazo, o cuando el embarazo se presenta durante perodos activos del Hodgkin, ambas entidades se ven agravadas seriamente en su evolución y por lo tanto el médico debe intervenir. En los países donde la religión y las leyes no permiten el aborto terapéutico de. be hacerse un tratamiento enérgico con radiaciones, teniendo siempre el cuidado de evitar no solo la irradación directa sino tam. bién la secundaria y de penumbra sobre el útero y su contenido, pues con ello pueden provocarse anomalias en el desarrollo fetal; por este mismo motivo debe también evitarse la administración de cualquiera de las drogas citotóxicas usualmente empleadas en el tratamiento de los linfomas.

Donde la interrupción del embarazo es permitida, entrarian en juego para decidir sobre su indicación factores múltiples como la edad de la paciente, el grado de diseminación de la enferme. dad, la edad del embarazo mismo, el número de hijos de la enferma. la mayor o menor importancia que para la familia y para la sociedad puedan tener la madre y $\in 1$ feto, respectivamente, etc. So lo la consideración juiciosa y desprevenida de estos factores, he. cha por el médico y por la familia de la paciente y a veces por la paciente misma puede definir la conducta en cada caso.

3) Cuando el embarazo se presenta en pacientes que están en período de inactividad del Hodgkin, parece que no hay reper. cusión sobre el estado de la paciente y que por lo tanto el emba. 
razo puede seguir su evolución normal. Las posibilidades de recaída de la enfermedad son las mismas que en cualquier otra mujer no embarazada

4) Según este último punto que mencionamos y bajo el aspecto netamente médico, no habría motivo para desaconsejar el em. barazo en las mujeres que han sido tratadas antes para enferme dad de Hodgkin y que en ese momento están en buenas condicio. nes. Sin embargo, nosotros creemos que sí hay una razón, y muy poderosa, para esta proscripción. Nos referimos al hecho demos. trado ya por las numerosas estadísticas que sobre el Hodgkin se han hecho en el mundo entero de que esta enfermedad es de evo_ lución muy desfavorable en la mayoría de los casos y que no pue. de considerarse como definitivamente curada ni siquiera después de haber pasado 5 años del tratamiento. Es decir, que aunque el €mbarazo no agrave a una paciente en período de inactividad, esa enferma está siempre expuesta a una recaída del Hodgkin en cualquier momento, que muy posiblemente la lleve a la muerte (las recidivas son siempre de peor pronóstico) y se agregaría en. tonces un huérfano más a los que ya pueda dejar esta mujer.

5) No existe ninguna evidencia de que los hijos de mujeres hodgkinianas tengan más posibilidades de presentar anomalías del desarrollo o estén más expuestos a la aparición de lesiones malignas.

\section{BIBLIOGRAFIA}

1. BICHEL, J.-Hodgkin's disease and pregnancy. Acta Radiol., 33:427, 1950.

2. GILBERT, R.-Grossesses au cours de lymphcgranulomes traités par roentgentherapic. Sort des meres et des enfants. Schw. med. Wschr., 75:53, 1945.

3. GILBERT, R.-The problem of pregnancy in Hodgkin's disease. Acta Radiol., 35:71, 1951.

4. GILBERT, R.-Lymphogranulomatose (Maladie de Hodgkin) ou granulomatose maligne. Capítulo IXXII en "Electroradiotherapie" de Delherm, pag. 1829. Masson et Cie. Paris. 1951.

5. HARTVIGSEN, F. B.-Hodgkin's disease and pregnancy. Acta Radiol., $44: 317,1955$.

6. HENNESSY, J. P. and ROTTINO, A.-Hodgkin's disease and pregnan. cy with report of twelve cases. Am. J. Obst. \& Gynec., 63: 756, 1952. 1954.

7. HULtbeRG, S.-Pregnancy in Hodgkin's disease. Acta Radiol., 41:277, 
8. PORTMANN, U. V. and MULEY, B. E.-Hodgkin's disease and pregzancy. Report of four cases. Cleveland Clin. Quart., 17:149, 1950.

9. RIVA, H. L., ANDRESON, P. S. and O'GRADY, J. W. Pregnancy and Hodgkin's disease; report of eight cases. Am. J. Obst. and Gynec., 66:866, 1953.

10. SOUTHAM, C. M., DIAMOND, H. D. and CRAVER, L. F. Pregnancy during Hodgkin's disease. Cancer, 9:1141, Oct_Dec., 1956.

11. TENEBLAT, W and HORTON, C. Hodgkin's disease and pregnancy. Review of literature and report of case. West. J. Surg., 59:120, 1951.

12. OTERO, E.-Dos observaciones sobre incidencia familiar de linfobrasto_ mas. Rev. Colombiana de Cancerol., 2:29, 1955.

13. GAITAN-YANGUAS, M.-Análisis de 253 casos de Enfermedad de Hodgkin. Trabajo leído en las IV Jornadas Radiológicas Colombianas. No_ viembre, 1957. En prensa en Unidia. 